\title{
A Mild Causal Relationship between Tea Consumption and Decreased Risk for Obesity in General Population: A Two-Sample Mendelian Randomization Study
}

\section{Cancan Li}

Shandong First Medical University - Tai'an Campus

\section{Mingyun Niu}

The Second Affiliated Hospital of Shandong First Medical University

\section{Zheng Guo}

Edith Cowan University

Pengcheng Liu

Shandong First Medical University - Tai'an Campus

\section{Yulu Zheng}

Edith Cowan University

Di Liu

Capital Medical University

\section{Song Yang}

Taian City Central Hospital

\section{Wei Wang}

Edith Cowan University

\section{Yuanmin Li}

The Second Affiliated Hospital of Shandong First Medical University

Haifeng Hou ( $\nabla$ hfhou@163.com )

Shandong First Medical University - Tai'an Campus

\section{Research}

Keywords: Tea consumption, Obesity, Mendelian Randomization analysis

Posted Date: April 6th, 2021

DOl: https://doi.org/10.21203/rs.3.rs-389585/v1

License: (c) (1) This work is licensed under a Creative Commons Attribution 4.0 International License. Read Full License 
Page 2/15 


\section{Abstract \\ Background}

Tea consumption is considered as a protective factor for obesity. This study aimed to verify the casual association between tea consumption and obesity through a two-sample Mendelian randomization (MR) analysis in general population-based datasets.

\section{Methods}

The genetic instruments, single nucleotide polymorphisms (SNPs) associated with tea consumption habits, were obtained from genome-wide association studies (GWAS): UK Biobank, Nurses' Health Study, Health Professionals Follow-up Study and Women's Genome Health Study. The effect of the genetic instruments on obesity was analyzed using UK Biobank dataset (among 500,000 participants). The causal relationship between tea consumption and obesity risk was analyzed by five methods of MR analyses: inverse variance weighted (IVW) method, MR-Egger regression method, weighted median estimator (WME), weighted mode and simple mode.

\section{Results}

Ninety-one SNPs were identified as genetic instruments in our study. A significant result was observed in IVW analysis (odds ratio [OR] $=0.998,95 \%$ confidence interval $[\mathrm{Cl}]=0.996$ to $1.000, P=0.049]$ ), which is the commonly used approach of two-sample MR analysis.

\section{Conclusion}

Our findings evidenced a mild causal relationship between tea consumption and the decreased risk for obesity. Further studies are needed to clarify the effects of tea consumption on obesity-related health problems in detail.

\section{Background}

Obesity is a nutrition-related metabolic disorder caused by genetic and environmental determinants [1, 2]. Obesity and obesity-related diseases have been public health burdens worldwide, especially in developed countries. In the United States, the healthcare expense was about $\$ 1,901$ per year for each obese person, which extrapolated to about $\$ 149,4$ billion at the national level [3]. Due to the continuous rise of incidence in the past 50 years, obesity has now reached pandemic proportion [2, 4], and is predicted to $20 \%$ by 2025 [5]. Moreover, obesity increases the risk of various severe complications, such as type 2 diabetes, cardiovascular disease, dementia and cancers [2]. In spite of the crucial role of diet and exercise in the treatment of obesity, supportive herbal remedies are of increasing concerns [6]. 
Tea is one of popular beverages globally, which is consumed up to 2 billion cups per day [7]. Tea is considered as an anti-obesity beverage attributed to three main components: tea polyphenols, tea polysaccharide and caffeine [8-10]. Although growing researches have focused on the relationship between tea and anti-obesity, the findings are inconsistent [11-14] .

Conventional epidemiological studies are susceptible to the potential confounders and inverse causality, which over- or under-estimate the causal relationship between determinants and outcomes. Mendelian randomization (MR) analysis is able to control the biases by introducing instrumental variables [15]. In MR studies, genetic variants that are closely associated with exposure factor are defined as instrumental variables, by which the causation between exposure factor and outcome is measured by genetic variants as substitution [16]. Since the formation of gametes follows the Mendelian law of "parental alleles randomly assigned to offspring", genetic variation is not affected by traditional confounding factors, and is associated with outcomes in a time-sequential manner [17]. In the current study, a two-sample MR analysis was used to assess the causal relationship between tea consumption and obesity in general population-based databases.

\section{Methods}

\section{Datasets}

For the database of exposure, significant single nucleotide polymorphisms (SNPs) related to tea consumption $\left(P<5 \cdot 10^{-8}\right)$ were obtained from a genome wide association study (GWAS) among 370,000 participants of European ancestry, which included participants in UK Biobank, Nurses' Health Study, Health Professionals Follow-up Study and Women's Genome Health Study [18]. The linkage disequilibrium (LD) of significant SNPs linked to tea consumption was set to meet $r^{2}<0.001$ to avoid the effect of strong LD on the results. The outcome datasets of obesity were obtained from the UK Biobank study which recruited about 500,000 European participants in 2006-2010. The relevant data were extracted from two databases respectively, including SNP sites, alleles, effect estimates for exposure and outcome, standard error (SE), and $P$ values.

\section{Statistical analysis}

There are three premises for two-sample MR [17, 19]: 1) Genetic variation as an instrumental variable must be closely related to exposure. 2) Instrumental variables are not associated with any known confounders. 3) The instrumental variables are not directly related to the outcome, that is, the instrumental variables cannot affect the outcome in other ways except through the exposure factors.

Prior to two-sample MR analysis, there is a need to unify the effect-value directions of exposure data and outcome data. Exposure and outcome data are unified into a dataset by removing the intermediate allele frequencies of SNPs containing palindromes [20]. In addition, SNPs with A/T or G/C alleles are defined as palindromic SNPs, "intermediate allele frequencies" referred to $0.01<$ allele frequency $<0.30$ [21]. 
Inverse variance weighted (IVW) method, MR-Egger regression method, weighted median estimator (WME), weighted mode and simple mode were used to evaluate the causal effect between tea consumption and obesity risk, and subsequently checked the stability and reliability of the results. The IVW model is a weighted linear regression model, which is based on the premise that all genetic variants are valid instrumental variables [22]. MR-Egger regression method can obtain unbiased estimation when there is pleiotropy in instrumental variables, measure average pleiotropy through intercept term, and perform sensitivity analysis [23]. WME can still calculate the causal association effect when the genetic variation below $50 \%$ violates the core assumptions of MR [24].

The intercept of the MR-Egger regression line illustrated the magnitude of the genetic pleiotropy. It was considered that there was no pleiotropic effect if no significant difference between intercept and $0(P>$ 0.05) [23]. Cochran's $Q$ statistic was used to assess the heterogeneity among the estimates from included SNPs. Funnel plot showed the relationship between the individual Wald ratio of each SNP and its accuracy, and whether its symmetry indicated whether the results had directional horizontal pleiotropy [25]. The "leave-one-out" method was used for sensitivity analysis. By gradually eliminating each SNP and calculating the combined effect of the remaining SNPs, the influence of individual SNP on results and the stability of the results were evaluated [26].

All data analyses were performed by the "TwoSampleMR" package in R version 4.0.2 (R Foundation for Statistical Computing, Vienna, Austria). Statistical significance was set as two-tailed $P<0.05$ unless otherwise specified.

\section{Results}

\section{Instrumental variable selection}

A total of 108 significant SNPs $\left(P<5 \times 10^{-8}\right.$, LD r $\left.^{2}<0.001\right)$ were obtained from the GWAS about tea consumption [18]. Among them 16 SNPs were removed for being palindromic with intermediate allele frequencies, and one SNP was removed because of no corresponding outcome data. Finally, 91 SNPs were selected to perform the following MR analysis. The detailed information of these SNPs was shown in Additional file 1: Table S1, mainly including effect allele (EA), other allele (OA) and summary statistics (beta coefficient, SE and $P$-value).

\section{MR analysis}

The causation between tea consumption and obesity was analyzed using the methods of IVW, MR Egger, WME, weighted mode and simple mode, independently. As shown in Table 1 and Fig. 1, a statistical significance was observed in IVW method analysis [odds ratio $(\mathrm{OR})=0.998,95 \%$ confidence interval $(\mathrm{Cl})$ $=0.996$ to $1.000, P=0.049$ ] (Table 1, Figs. 1 and 2). No significant causal relationships were observed in the analyses of MR Egger $(\mathrm{OR}=1.003,95 \% \mathrm{Cl}=0.998$ to $1.008, P=0.255)$, WME $(\mathrm{OR}=0.998,95 \% \mathrm{Cl}=$ 0.996 to $1.001, P=0.262)$, weighted mode (OR $=0.999,95 \% \mathrm{Cl}=0.994$ to $1.003, P=0.505)$ or simple mode $(\mathrm{OR}=0.999,95 \% \mathrm{Cl}=0.993$ to $1.005, P=0.747)($ Table 1 , Figs. 1 and 2$)$. 
Table 1

Two-sample Mendelian Randomization for tea consumption on obesity risk

\begin{tabular}{|llllll|}
\hline Method & N SNPs & Beta coefficient & SE & OR (95\%Cl) & $P$ \\
\hline IVW & 91 & -0.002 & 0.001 & $0.998(0.996-1.000)$ & 0.049 \\
\hline MR-Egger & 91 & 0.003 & 0.003 & $1.003(0.998-1.008)$ & 0.255 \\
\hline WME & 91 & -0.002 & 0.001 & $0.998(0.996-1.001)$ & 0.262 \\
\hline Weighted mode & 91 & -0.001 & 0.002 & $0.999(0.994-1.003)$ & 0.505 \\
\hline Simple mode & 91 & -0.001 & 0.003 & $0.999(0.993-1.005)$ & 0.747 \\
\hline $\begin{array}{l}\text { N SNPs indicates the number of single nucleotide polymorphisms; SE, standard error; OR, odds ratio; } \\
\text { Cl, confidence interval; IVW, inverse variance weighted; WME, weighted median estimator. }\end{array}$ \\
\hline
\end{tabular}

\section{Sensitivity analysis}

The leave-one-out method displayed that the results of two-sample MR analysis were strong (Fig. 3), indicating that no instrumental variables influenced the causal inference. No significant heterogeneity was found across the estimates of included SNPs (Cochran's $Q=86.692, P=0.550$ ). The funnel plot analysis showed a symmetry result (Fig. 4), by which non-significance in directional and horizontal multipolarity was observed. In addition, genetic pleiotropy test was carried out by the MR-Egger regression analysis. A non-significant intercept was obtained [intercept=-5.885727e-05 (SE $=2.995104 \mathrm{e}-$ $05), P=0.053]$ in this test, illustrating that our findings were not influenced by the polymorphisms.

\section{Discussion}

Our two-sample MR analyses are conducted in five independent approaches. The result of IVW analysis evidenced that tea consumption has a mild causal relationship with decreased risk for obesity in general population. The results of MR Egger, WME, weighted mode and simple mode analyses did not show causal relationship between tea and obesity. Nevertheless, IVW is the most widely used and usually provides predominant results $[22,27]$. The results of sensitivity analyses showed no statistical difference, which proved that these two-sample MR results were reliable.

As a popular, economical and safe drink, the effect of tea on anti-obesity is widely understood, especially among overweight and obese individuals [8-10,28]. The potential mechanisms of tea on anti-obesity are as follows: 1) reducing food intake and energy absorption [29], 2) regulating the expression of lipid metabolism genes and inhibiting fat accumulation [30],3) enhancing the activity of antioxidant defense enzymes [31, 32], 4) regulating intestinal microflora disturbance and attenuating intestinal inflammation $[33,34]$, and 5) maintaining intestinal barrier integrity [35].

In recent years, a growing number of studies have begun to explore the association between tea consumption and obesity. Two reviews showed no-significant link between tea and weight loss in obese 
people $[11,12]$. On the other hand, some randomized controlled trials (RCTs) proved the anti-obesity effect of tea on obesity [36-38]. In addition, two meta-analyses published in 2020 came up with similar results $[13,14]$. Our study, a two-sample MR analysis based on general population-based datasets, verified the casual relationship between tea consumption and decreased obesity risk.

Traditional epidemiological studies, consisting of case-control studies and cohort studies, provide representative findings on the relationship between exposures and outcomes. However, these studies are usually biased by confounding factors and adverse causal effects [39-41]. MR analysis can control the biases by introducing instrumental variables $[25,42]$. MR analysis on general population-based datasets is a novel approach to provide evidence on causation. Our MR study based on UK Biobank, Nurses' Health Study, Health Professionals Follow-up Study and Women's Genome Health Study validated that tea consumption have a mild causal relationship with decreased risk for obesity.

\section{Limitations}

There are inevitable limitations that should be notified. First, potential horizontal pleiotropy could not be comprehensively assessed even though multiple sensitivity analyses were performed. However, Cochran's Q statistic and MR-Egger intercept test found that there were no heterogeneity or pleiotropy in this MR analysis. Second, we did not carry out subgroup analysis due to the lack of demographic information in detail. In addition, the study population were of European ancestry, which might lead to ethic bias.

\section{Conclusion}

Our findings evidenced that tea consumption has a mild causal relationship with decreased risk for obesity in general population. More studies are needed to clarify the effects of tea and its components on obesity-related health problems.

\section{Abbreviations}

MR

Mendelian randomization, SNPs:nucleotide polymorphisms, GWAS:genome-wide association studies, LD:linkage disequilibrium, IVW:inverse variance weighted, WME:weighted median estimator, OR:odds ratio, Cl:confidence interval, EA:effect allele, OA:other allele.

\section{Declarations}

\section{Ethics approval and consent to participate}

Not applicable.

\section{Consent for publication}


Not applicable.

\section{Availability of data and materials}

The data of obesity is available at http://www.nealelab.is/uk-biobank.

\section{Competing interest}

The authors declare no conflict of interest.

\section{Funding}

This study was supported by the Shandong Provincial Natural Science Foundation, China [ZR2017MH100, ZR2017MH097], and Australia-China Collaborative Grant [NHMRC APP1112767-NSFC 81561128020].

\section{Author Contributions}

C.L. did the data analysis and explained the results, C.L., M.N. and Z.G. wrote the manuscript., P.L., Y.Z., D.L., S.Y., and W.W. reviewed manuscript. Y.L. and H.H. decided the idea of the research and were responsible for the whole research. All authors had read and approved the final manuscript.

\section{Acknowledgments}

We thank all researchers for sharing the GWAS pooled data on tea consumption and obesity risk.

\section{References}

1. González-Muniesa P, Mártinez-González MA, Hu FB, et al. Obesity. Nature reviews Disease primers. 2017;3:17034.

2. Blüher M. Obesity: global epidemiology and pathogenesis. Nature reviews Endocrinology. 2019;15(5):288-98.

3. Kim DD, Basu A. Estimating the Medical Care Costs of Obesity in the United States: Systematic Review, Meta-Analysis, and Empirical Analysis. Value in health: the journal of the International Society for Pharmacoeconomics Outcomes Research. 2016;19(5):602-13.

4. Chooi YC, Ding C, Magkos F. The epidemiology of obesity. Metabolism: clinical experimental. 2019;92:6-10.

5. (NCD-RisC) NRFC. Trends in adult body-mass index in 200 countries from 1975 to 2014: a pooled analysis of 1698 population-based measurement studies with $19 \cdot 2$ million participants. Lancet (London England). 2016;387(10026):1377-96.

6. Liang Y, Lin C, Huang S, Xu Y. Traditional Chinese Medicine and Intestinal Microbiota: A Complementary and Integrative Health Approach to Ameliorate Obesity-Related Diseases. Holistic nursing practice. 2019;33(5):259-65. 
7. Drew L. The growth of tea. Nature. 2019;566(7742):2-s4.

8. Wang S, Moustaid-Moussa N, Chen L, et al. Novel insights of dietary polyphenols and obesity. The Journal of nutritional biochemistry. 2014;25(1):1-18.

9. Xu Y, Zhang M, Wu T, Dai S, Xu J, Zhou Z. The anti-obesity effect of green tea polysaccharides, polyphenols and caffeine in rats fed with a high-fat diet. Food function. 2015;6(1):297-304.

10. Chen G, Xie M, Wan P, et al. Fuzhuan Brick Tea Polysaccharides Attenuate Metabolic Syndrome in High-Fat Diet Induced Mice in Association with Modulation in the Gut Microbiota. Journal of agricultural food chemistry. 2018;66(11):2783-95.

11. Jurgens TM, Whelan AM, Killian L, Doucette S, Kirk S, Foy E. Green tea for weight loss and weight maintenance in overweight or obese adults. The Cochrane database of systematic reviews. 2012;12:Cd008650.

12. Baladia E, Basulto J, Manera M, Martínez R, Calbet D. [Effect of green tea or green tea extract consumption on body weight and body composition; systematic review and meta-analysis]. Nutricion hospitalaria. 2014;29(3):479-90.

13. Li X, Wang W, Hou L, et al. Does tea extract supplementation benefit metabolic syndrome and obesity? A systematic review and meta-analysis. Clinical nutrition (Edinburgh Scotland). 2020;39(4):1049-58.

14. Lin Y, Shi D, Su B, et al. The effect of green tea supplementation on obesity: A systematic review and dose-response meta-analysis of randomized controlled trials. Phytotherapy research: PTR. 2020;34(10):2459-70.

15. Cao W, Li X, Zhang X, et al. No Causal Effect of Telomere Length on Ischemic Stroke and Its Subtypes: A Mendelian Randomization Study. Cells. 2019;8(2).

16. Zhang X, Tian Q, Liu D, et al. Causal association of circulating cholesterol levels with dementia: a mendelian randomization meta-analysis. Translational psychiatry. 2020;10(1):145.

17. Emdin CA, Khera AV, Kathiresan S. Mendelian Randomization. Jama. 2017;318(19):1925-6.

18. Zhong VW, Kuang A, Danning RD, et al. A genome-wide association study of bitter and sweet beverage consumption. Human molecular genetics. 2019;28(14):2449-57.

19. Greenland S. An introduction to instrumental variables for epidemiologists. International journal of epidemiology. 2018;47(1):358.

20. Hemani G, Zheng J, Elsworth B, et al. The MR-Base platform supports systematic causal inference across the human phenome. elife. 2018;7.

21. Hartwig FP, Davies NM, Hemani G, Davey Smith G. Two-sample Mendelian randomization: avoiding the downsides of a powerful, widely applicable but potentially fallible technique. International journal of epidemiology. 2016;45(6):1717-26.

22. Yuan S, Kar S, Vithayathil M, et al. Causal associations of thyroid function and dysfunction with overall, breast and thyroid cancer: A two-sample Mendelian randomization study. International journal of cancer. 2020;147(7):1895-903. 
23. Bowden J, Davey Smith G, Burgess S. Mendelian randomization with invalid instruments: effect estimation and bias detection through Egger regression. International journal of epidemiology. 2015;44(2):512-25.

24. Bowden J, Davey Smith G, Haycock PC, Burgess S. Consistent Estimation in Mendelian Randomization with Some Invalid Instruments Using a Weighted Median Estimator. Genetic epidemiology. 2016;40(4):304-14.

25. Choi Y, Lee SJ, Spiller W, et al. Causal Associations Between Serum Bilirubin Levels and Decreased Stroke Risk: A Two-Sample Mendelian Randomization Study. Arteriosclerosis thrombosis vascular biology. 2020;40(2):437-45.

26. Mokry LE, Ross S, Timpson NJ, Sawcer S, Davey Smith G, Richards JB. Obesity and Multiple Sclerosis: A Mendelian Randomization Study. PLoS medicine. 2016;13(6):e1002053.

27. Dan YL, Wang P, Cheng Z, et al. Circulating adiponectin levels and systemic lupus erythematosus: a two-sample Mendelian randomization study. Rheumatology (Oxford, England). 2020.

28. Ahmad RS, Butt MS, Sultan MT, et al. Preventive role of green tea catechins from obesity and related disorders especially hypercholesterolemia and hyperglycemia. Journal of translational medicine. 2015;13:79.

29. Yang CS, Zhang J, Zhang L, Huang J, Wang Y. Mechanisms of body weight reduction and metabolic syndrome alleviation by tea. Molecular nutrition food research. 2016;60(1):160-74.

30. Chen LH, Chien YW, Liang CT, Chan CH, Fan MH, Huang HY. Green tea extract induces genes related to browning of white adipose tissue and limits weight-gain in high energy diet-fed rat. Food nutrition research. 2017;61(1):1347480.

31. Ren D, Hu Y, Luo Y, Yang X. Selenium-containing polysaccharides from Ziyang green tea ameliorate high-fructose diet induced insulin resistance and hepatic oxidative stress in mice. Food function. 2015;6(10):3342-50.

32. Chen $G$, Wang $M$, Xie M, et al. Evaluation of chemical property, cytotoxicity and antioxidant activity in vitro and in vivo of polysaccharides from Fuzhuan brick teas. International journal of biological macromolecules. 2018;116:120-7.

33. Zhou C, Zhou X, Wen Z, et al. Compound Fu brick tea modifies the intestinal microbiome composition in high-fat diet-induced obesity mice. Food science nutrition. 2020;8(10):5508-20.

34. Li Y, Rahman SU, Huang Y, et al. Green tea polyphenols decrease weight gain, ameliorate alteration of gut microbiota, and mitigate intestinal inflammation in canines with high-fat-diet-induced obesity. The Journal of nutritional biochemistry. 2020;78:108324.

35. Lu X, Liu J, Zhang N, et al. Ripened Pu-erh Tea Extract Protects Mice from Obesity by Modulating Gut Microbiota Composition. Journal of agricultural food chemistry. 2019;67(25):6978-94.

36. Chen IJ, Liu CY, Chiu JP, Hsu CH. Therapeutic effect of high-dose green tea extract on weight reduction: A randomized, double-blind, placebo-controlled clinical trial. Clinical nutrition (Edinburgh Scotland). 2016;35(3):592-9. 
37. Brown AL, Lane J, Holyoak C, Nicol B, Mayes AE, Dadd T. Health effects of green tea catechins in overweight and obese men: a randomised controlled cross-over trial. The British journal of nutrition. 2011;106(12):1880-9.

38. Huang LH, Liu CY, Wang LY, Huang CJ, Hsu CH. Effects of green tea extract on overweight and obese women with high levels of low density-lipoprotein-cholesterol (LDL-C): a randomised, double-blind, and cross-over placebo-controlled clinical trial. BMC complementary alternative medicine. 2018;18(1):294.

39. Doherty C, Delahunt E, Caulfield B, Hertel J, Ryan J, Bleakley C. The incidence and prevalence of ankle sprain injury: a systematic review and meta-analysis of prospective epidemiological studies. Sports medicine (Auckland NZ). 2014;44(1):123-40.

40. Richardson K, Fox C, Maidment I, et al. Anticholinergic drugs and risk of dementia: case-control study. BMJ. 2018;361:k1315.

41. Erfani $\mathrm{H}$, Haeri S, Shainker SA, et al. Vasa previa: a multicenter retrospective cohort study. American journal of obstetrics and gynecology. 2019;221(6):644.e641-644.e645.

42. Gage SH, Bowden J, Davey Smith G, Munafò MR. Investigating causality in associations between education and smoking: a two-sample Mendelian randomization study. International journal of epidemiology. 2018;47(4):1131-40.

\section{Figures}




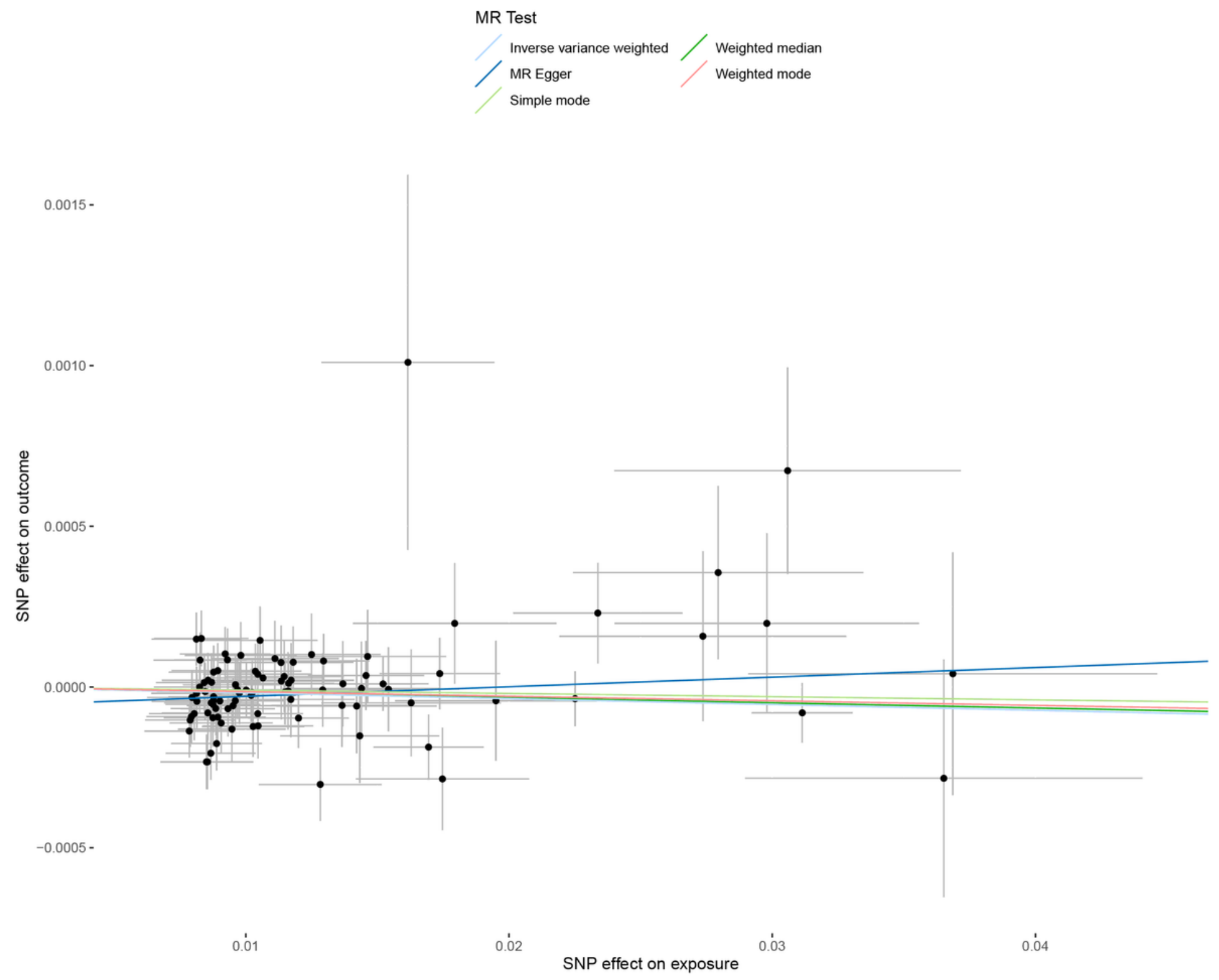

Figure 1

Scatter plot to visualize the causal effect between tea consumption and obesity risk. The slope of the straight line indicates the magnitude of the causal association, scatter plot of inverse variance weighted (IVW) method, MR-Egger regression method, weighted median estimator (WME), weighted mode and simple mode. MR, Mendelian randomization; SNP, single nucleotide polymorphism. 


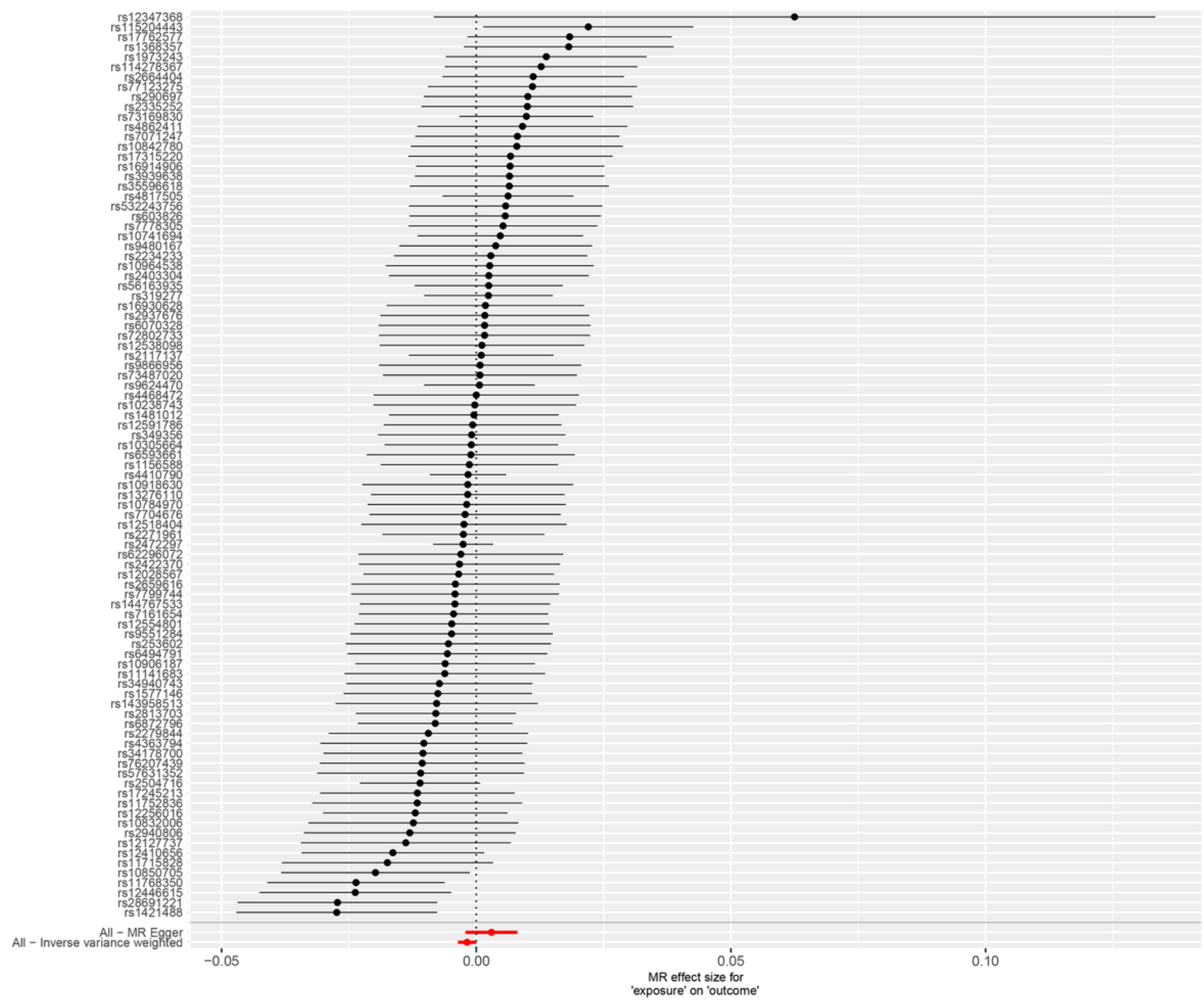

Figure 2

Forest plot to show the casual effect of tea consumption on obesity risk. Forest plot of IVW and MR-Egger regression method. MR, Mendelian randomization. 


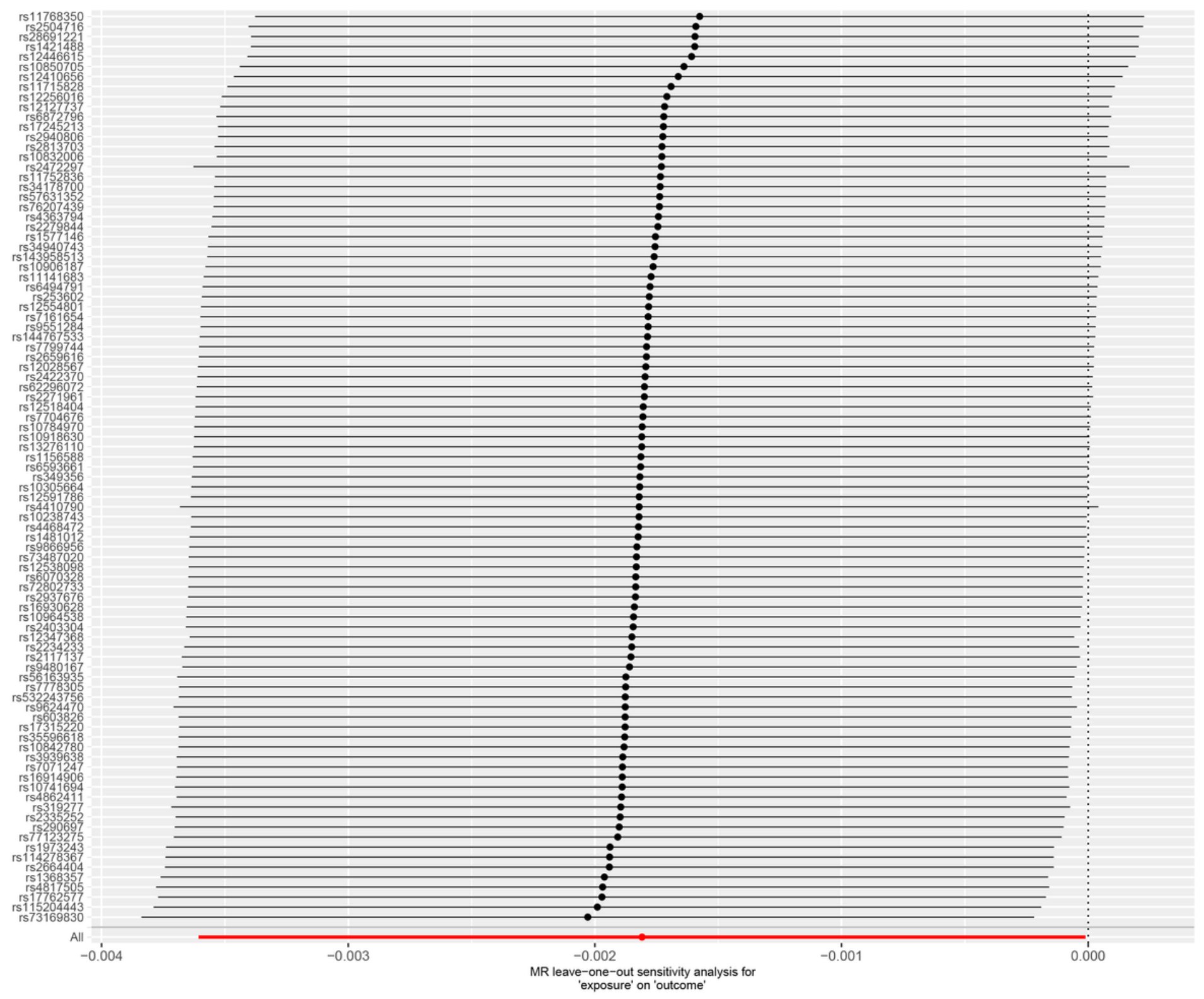

\section{Figure 3}

Forest plot of "leave-one-out" sensitivity analysis method to show the influence of individual SNP on the results. MR, Mendelian randomization. 


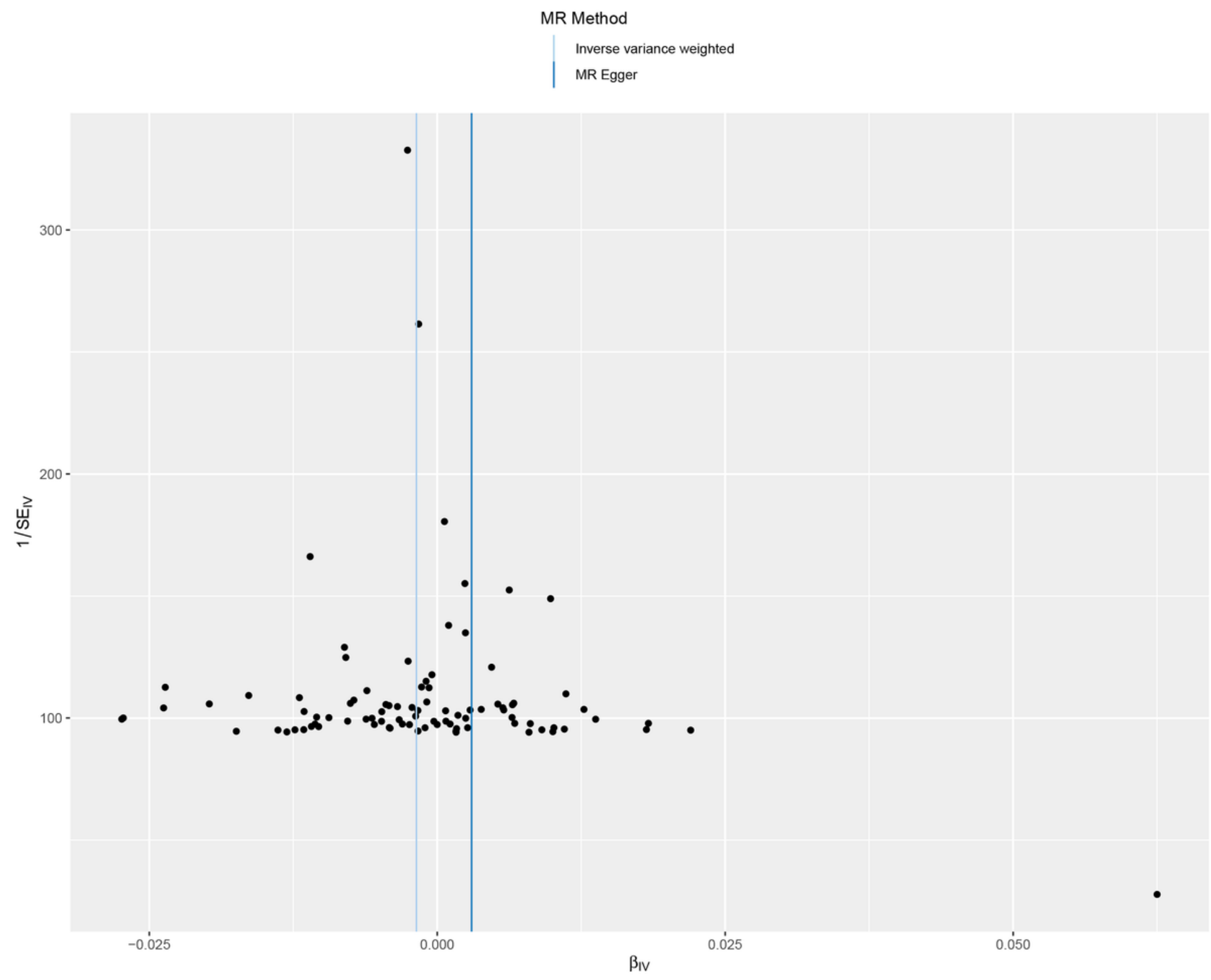

Figure 4

Funnel plot to visualize overall heterogeneity of Mendelian randomization assessment for the effect of tea consumption on obesity risk. MR, Mendelian randomization; SE, standard error; IV, instrument variable.

\section{Supplementary Files}

This is a list of supplementary files associated with this preprint. Click to download.

- Additionalfile1.docx 